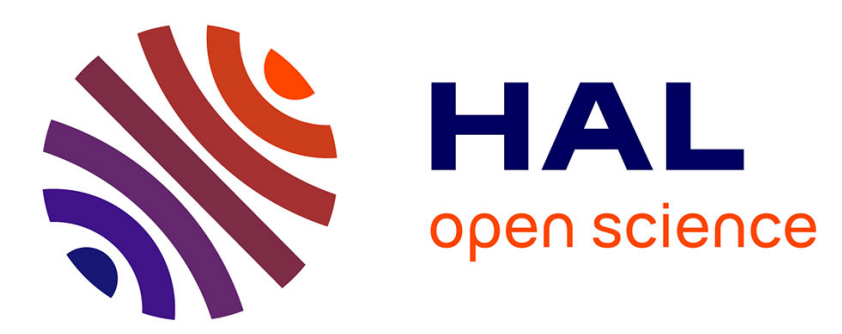

\title{
Une méthode de calibration simultanée des accéléromètres et des pots vibrants dans le plan transversal
}

\author{
C.-A. Faure, Xavier Boutillon
}

\section{- To cite this version:}

C.-A. Faure, Xavier Boutillon. Une méthode de calibration simultanée des accéléromètres et des pots vibrants dans le plan transversal. Journal de Physique IV Proceedings, 1994, 04 (C5), pp.C5-267-C5270. 10.1051/jp4:1994554 . jpa-00253049

\section{HAL Id: jpa-00253049 https://hal.science/jpa-00253049}

Submitted on 1 Jan 1994

HAL is a multi-disciplinary open access archive for the deposit and dissemination of scientific research documents, whether they are published or not. The documents may come from teaching and research institutions in France or abroad, or from public or private research centers.
L'archive ouverte pluridisciplinaire HAL, est destinée au dépôt et à la diffusion de documents scientifiques de niveau recherche, publiés ou non, émanant des établissements d'enseignement et de recherche français ou étrangers, des laboratoires publics ou privés. 


\title{
Une méthode de calibration simultanée des accéléromètres et des pots vibrants dans le plan transversal
}

\author{
C.-A. FAURE et X. BOUTILLON
}

Laboratoire d'Acoustique Musicale, CNRS, Université Paris 6, Ministère de la Culture, Boîte Courrier 161, 4 place Jussieu, 75252 Paris cedex 05, France

\begin{abstract}
The cross-axis sensitivity of accelerometers that is of the order of a few hundredths of the main axis sensitivity, is a common concern in vibration measurements. Usually the manufacturers do not provide a complete information upon the transverse sensitivity of transducers. The transverse motion of mechanical shakers is subject to the same problem and lack of information. The method that we present here is aimed at calibrating at the same time both defaults relatively to the axial components. By mounting an accelerometer on a shaker so that the main motion is perpendicular to the main sensitivity, its response adds up two products of the same order of magnitude : the main motion $x$ the cross-axis sensitivity and the main sensitivity $x$ the transverse motion. These two quantities are separated when the accelerometer rotates around the main-motion axis. All relative components of transverse motion and cross-axis sensitivity are derived from a combination of orientations of the accelerometer relatively to the main-motion axis. Measurements made with a variety of accelerometers exhibit a good consistency of the results concerning the transverse motion of a given shaker. Conversely, a good reproducibility of results pertaining to the cross-axis sensitivity of accelerometers has been achieved. The range in which that method is easily used with good results is limited in the low-frequency region by the motion of the shaker base and attachment.
\end{abstract}

\section{1 - INTRODUCTION}

Pour tout accéléromètre l'existence d'une sensibilité transversale se superposant à la sensibilité dans l'axe principal (ordinairement dans un rapport de quelques pour cents) peut poser des problèmes importants à l'expérimentateur [1]. Les données fournies par les constructeurs ne permettent généralement pas de prendre en compte cette sensibilité transversale. Sur les pots vibrants, la partie mobile peut avoir un mouvement transversal de l'ordre de quelques centièmes du mouvement dans l'axe principal et on se heurte à la même incertitude. Il existe évidemment des méthodes d'étalonnage utilisant des sources d'excitations mécaniques ou des accéléromètres étalons. La méthode exposée dans la suite permet d'étalonner simultanément les défauts d'alignements d'un pot vibrant et d'un accéléromètre.

\section{2 - PRINCIPE DE LA MÉTHODE}

\section{1 - Analyse vectorielle du problème}

L'hypothèse de linéarité de la réponse de l'accéléromètre permet de définir à chaque fréquence $f$ un unique vecteur sensibilité $S(f)$ tel que, quelque soit le vecteur $A$, la réponse, notée $R$, à une accélération de la forme A $\sin 2 \pi f t$ s'écrit :

$$
R=S(f) \cdot A \sin 2 \pi f t \quad(I)
$$

Par la suite la dépendance temporelle de la réponse de l'accéléromètre n'est plus indiquée. Dans le référentiel lié à l'accéléromètre, noté $(O X, O Y, O Z)$, le vecteur $S(f)$ peut être représenté par ses coordonnées 
$S_{1}, S_{2}, S_{3}$ dans le repère associé. L'axe $O Z$ est choisi de manière à ce qu'il définisse la direction de sensibilité principale de l'accéléromètre. $S_{1}$ et $S_{2}$ sont donc les coordonnées de la sensibilité transversale; elles restent faibles devant $S_{3}$.

Nous pouvons de la même manière définir pour le pot vibrant un référentiel lié à celui-ci, noté $(O x, O y, O z)$, $O z$ étant l'axe principal du pot vibrant. Dans le repère associé, nous notons $A_{1}, A_{2}, A_{3}$ les coordonnées de l'accélération du pot vibrant. On aura de même $A_{1}$ et $A_{2}$, petits devant $A_{3}$.

\section{2 - Réponse de l'accéléromètre}

On place l'accéléromètre dans une position arbitraire. On fait tourner l'accéléromètre autour de l'axe principal du pot $\mathrm{Oz}$. Les mesures obtenues tracent une sinusoïde dont la valeur moyenne ne dépend que des composantes de l'accélération du pot et de la sensibilité de l'accéléromètre selon cet axe $O z$. L'amplitude et la phase de la sinusoïde donnent des indications sur les autres composantes. En combinant diverses orientations de l'accéléromètre, on peut ainsi avoir accès à toutes les composantes transversales de la sensibilité et de l'accélération rapportées à leurs composantes principales.

\subsection{1 - Détermination de $S_{3}$}

Nous plaçons l'accéléromètre de telle manière que $O X=O x, O Y=O y$ et $O Z=O z$, puis nous le faisons tourner autour de $O z$ d'un angle $\theta(\theta=(O x, O X))$. La réponse de l'accéléromètre en fonction de $\theta$ s'écrit :

$$
R_{1}(\theta)=\left[S_{1} \cos (\theta)-S_{2} \sin (\theta)\right] A_{1}+\left[S_{1} \sin (\theta)+S_{2} \cos (\theta)\right] A_{2}+S_{3} A_{3}
$$

La valeur moyenne de $R_{1}(\theta)$ est égale au produit de $S_{3}$ et de $A_{3}$. La fonction sinusoïdale de $\theta$ est du second ordre devant le terme constant et il est difficile d'en obtenir, en pratique, des renseignements sur les quantités transversales.

\subsection{2 - Détermination de $S_{1}$}

On procède comme précédemment avec $O X=-O z, O Y=-O y$ et $O Z=-O x$, puis $\theta=(O x,-O Z)$. La réponse de l'accéléromètre en fonction de $\theta$, en négligeant les termes du second ordre devant ceux du premier ordre, s'écrit :

$$
R_{2}(\theta)=-S_{1} A_{3}-S_{3}\left[A_{1} \cos (\theta)+A_{2} \sin (\theta)\right]
$$

Ici le terme constant $-S_{1} A_{3}$ et le terme sinusoïdal sont du même ordre de grandeur (cf. fig.2). Les termes constants de (2) et (3) donnent accès à $S_{1} / S_{3}$. En décomposant la composante sinusoïdale sur les fonctions cosinus et sinus de $\theta$ et en divisant par la partie constante de (2) nous en déduisons l'amplitude relative selon les deux axes $O x$ et $O y$ du mouvement transversal du pot vibrant.

\section{3 .3 - Détermination de $S_{2}$}

De même avec ici $O X=-O y, O Y=-O z$ et $O Z=O x$, puis $\theta=(O x, O Z)$, la réponse de l'accéléromètre, en négligeant les termes du second ordre devant ceux du premier ordre, s'écrit:

$$
R_{3}(\theta)=-S_{2} A_{3}+S_{3}\left[A_{1} \cos (\theta)+A_{2} \sin (\theta)\right]
$$

Le terme constant $-S_{2} A_{3}$ et le terme sinusoïdal sont du même ordre de grandeur. Les termes constants de (2) et (4) donnent accès à $S_{2} / S_{3}$. Quant à la composante sinusoïdale, elle est l'opposée au second ordre près, de celle de (3), ce qui constitue un bon moyen de vérification de la méthode.

\section{3 - PROCÉDURE EXPERIMENTALE}

\section{1- Contraintes pratiques}

En pratique deux contraintes sont à respecter. En premier lieu, comme le mouvement du pot est influencé (même faiblement) par sa charge, la rotation de l'accéléromètre autour de $O z$ ne doit pas modifier de manière sensible le mouvement transversal du pot vibrant. D'autre part, au cours de la procédure permettant de mesurer les coordonnées transversales de la sensibilité de l'accéléromètre, l'angle entre $O z$ et l'axe du référentiel de l'accéléromètre avec lequel il coïncide en théorie (par exemple -OX pour $S_{I}$ voir 2.3.2) doit toujours rester de l'ordre du dixième de degré, faute de quoi l'influence de $S_{3}$ sur les résultats serait de l'ordre des quantités mesurées.

\section{2 - Dispositif mécanique expérimental}

Pour satisfaire ces contraintes l'accéléromètre est monté sur un support mécanique en trois pièces (cf. fig.

1). L'accéléromètre est collé sur un cube pouvant être fixé sur un plateau cylindrique selon trois faces 
différentes (perpendiculaires deux à deux). L'erreur d'angle évoquée ci-dessus est donc la superposition des défauts d'angles de la pièce cubique (contrôlable avec une précision supérieure au dixième de degré) et du défaut de collage. Le plateau cylindrique est en deux pièces imbriquées, la pièce supérieure pouvant tourner par rapport au pot autour de l'axe $O z$, alors que la pièce inférieure est fixée sur la partie mobile du pot vibrant. Dans la pièce supérieure trois trous filetés permettent de fixer le cube selon trois faces différentes en maintenant l'accéléromètre dans le prolongement de l'axe du pot vibrant $O z$. D'autre part 4 trous, auxquels correspondent 12 trous filetés dans le plateau inférieur permettent de fixer les deux pièces entre elles dans 12 positions différentes (correspondant à un pas de $30^{\circ}$ ).

Pour que le mouvement du pot ne soit pas trop sensible à la rotation du cube autour de l'axe Oz et donc à la modification de charge induite, la masse du plateau est bien supérieure à celles cumulées du cube et de l'accéléromètre.

\section{3 - Saisie des mesures : excitation, amplification, filtrage}

Le pot vibrant (B\&K 4810) est alimenté par une tension sinusoïdale à $400 \mathrm{~Hz}$ délivrée par un générateur, puis amplifiée. La tension appliquée au pot est visualisée à l'oscilloscope et vérifiée avant, et après chaque modification de la position de l'accéléromètre à l'aide d'un multimètre numérique. Le signal en sortie de l'accéléromètre est amplifié par un amplificateur de charge B\&K 2635, puis filtré autour de la composante fréquentielle qui nous intéresse $(400 \mathrm{~Hz})$. Il est ensuite mesuré à l'aide d'un multimètre numérique et visualisé à l'oscilloscope pour connaître le signe de la réponse de l'accéléromètre.

\section{4 - RÉSULTATS}

\section{1 - Résultats pour un accéléromètre}

La méthode exposée ci-dessus a été expérimentée sur un accéléromètre B\&K 4344 pour une excitation sinusoïdale de fréquence $400 \mathrm{~Hz}$. Sur la figure 2 sont tracées les mesures de $R_{2}(\theta)$ et $R_{3}(\theta)$. Comme les équations (3) et (4) le laissaient prévoir, les sinusoïdes de la figure 2 ont même amplitude et sont en opposition de phase. La mesure de la composante alternative de $R_{l}(\theta)$ est inexploitable, les incertitudes sur la tension appliquée au pot vibrant aussi bien que sur la mesure du signal en sortie d'amplificateur de charge sont de l'ordre de l'amplitude de la sinusoïde de (2).

\section{2 - Reproductibilité des mesures pour un accéléromètre}

Pour le même accéléromètre, sont représentées dans le plan transversal (cf. fig. 3) trois mesures de la sensibilité transversale, à la même fréquence $(400 \mathrm{~Hz})$ et pour trois collages différents. Nous pouvons constater une bonne reproductibilité de la méthode, les écarts maximums observés sur les mesures du vecteur de sensibilité transversale ne sont que de $10^{\circ}$ en angle et de $1 \%$ de la sensibilité principale en amplitude. Ces résultats sont une preuve à posteriori que le collage introduit des défauts d'angle inférieurs à celui due à l'existence d'une sensibilité transversale.

\section{3 - Reproductibilité des mesures pour le pot vibrant}

La reproductibilité des mesures du mouvement transversal du pot vibrant est vérifiée à partir de deux accéléromètres différents B\&K 4344 et 4374 à une fréquence de $400 \mathrm{~Hz}$ (cf. fig. 4). On observe une bonne concordance entre les deux résultats, les écarts entre les deux mesures du mouvement transversal étant de $5^{\circ}$ en angle et de $0,5 \%$ du mouvement dans l'axe principal en amplitude.

La méthode décrite ci-dessus s'applique dans une large bande de fréquence. Toutefois, en basse fréquence, le mouvement de la partie mobile du pot met en vibration de manière importante le support, surtout dans le plan horizontal. Comme le mouvement décrit par l'accéléromètre est celui défini dans le référentiel du laboratoire et non dans celui lié à la partie fixe du pot vibrant, cette vibration du support, très dépendante de la position du pot, représente une source notable d’incertitude sur le résultat.

\section{5 - CoNClusion}

Nous avons pu constater que la méthode présentée ici donne d'excellents résultats, aussi bien pour la sensibilité transversale que pour le mouvement transversal. Elle ne nécessite qu'un appareillage d'emploi courant en laboratoire. La réalisation du support de l'accéléromètre en trois pièces aux normes définies ne présente aucune difficulté à l'usinage. Le problème du support du pot peut être résolu en fixant le pot vibrant, ce qui permettra de confronter nos résultats à ceux obtenus par d'autres méthodes.

\section{6 - REMERCIEMENTS}


Les auteurs remercient les professeurs Gabriel Wenreich et Noël Perkins de l'Université du Michigan, ainsi que le Ministère de la Culture pour son aide financière.

\section{7 - RÉFÉRENCE}

[1] A.E. Bozhko, V.P. Shpachuck, V.I. Belykh 1985 Measurement techniques, 28 (7), 629-631. Measuring errors of amplitude and phase for vibratory accelerations when testing objects.

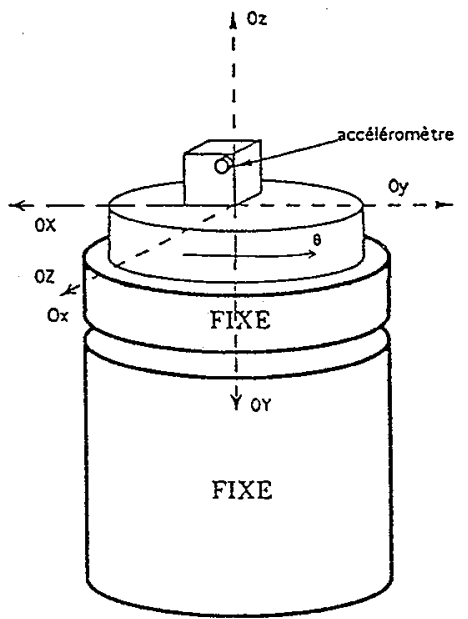

Fig.1 : Pot vibrant et pièce pour monter et faire toumer l'accéléromètre sans modifier la charge du pot.

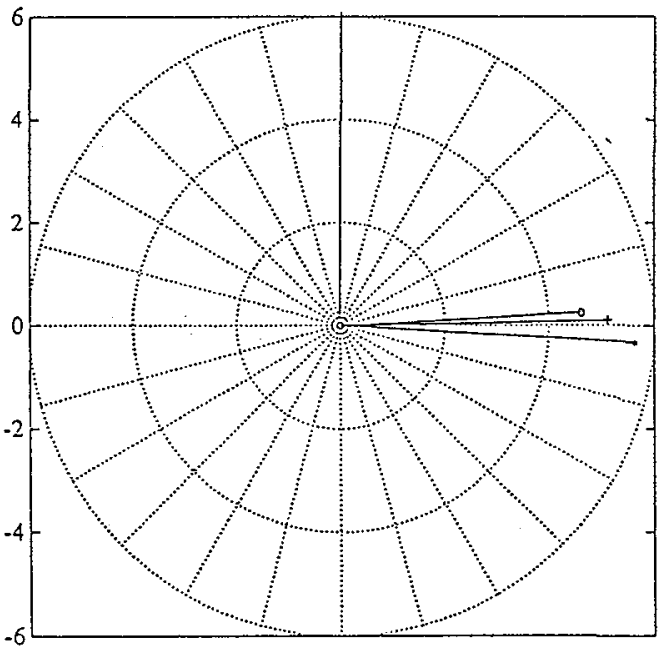

Fig. 3 : Trois mesures du vecteur sensibilité transversale d'un accéléromètre B\&K 4344 à $400 \mathrm{~Hz}$ rapportées à la sensibilité principale. Les vecteurs sont tracés dans le plan $O X Y$, le trait noir vertical indiquant un repère fixe sur l'accéléromètre.

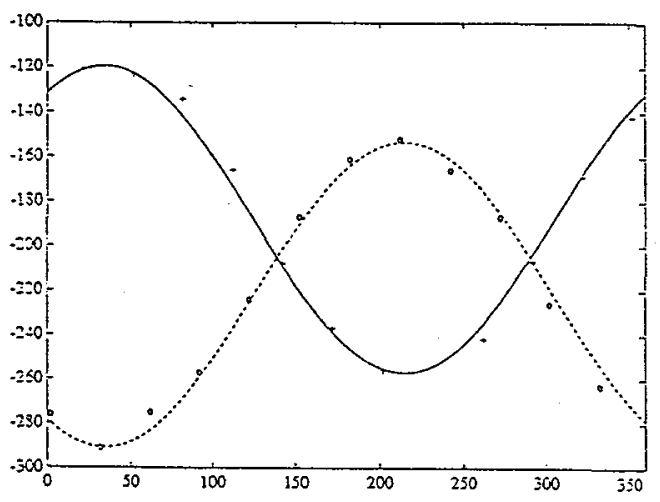

Fig.2 : Mesures de $R_{2}(\theta)$ (indiquées par des "+") et de $R_{3}(\theta)$ ("o") en $m V$ tracées en fonction de $\theta$ (exprimés en ${ }^{\circ}$ ) pour un accéléromètre $\mathrm{B} \& \mathrm{~K} 4344$ à $400 \mathrm{~Hz}$.

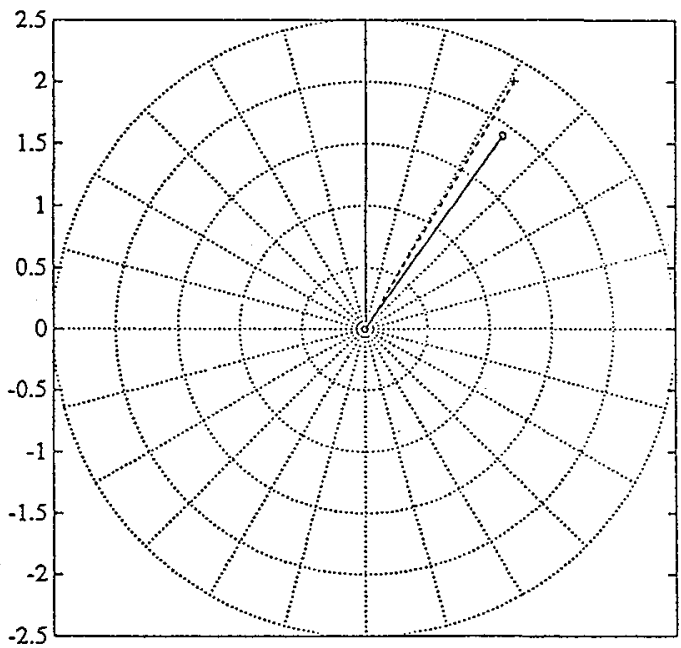

Fig. 4 : Deux mesures du mouvement transversal d'un pot vibrant B\&K 4810 à $400 \mathrm{~Hz}$ réalisées successivement à l'aide de deux accéléromètres . Les vecteurs sont rapportés au mouvement principal du pot et sont tracés dans le plan $O x y$ (le trait noir vertical indique un repère fixe sur le pot). 